Trauma Berufskrankh 2010 · 12[Suppl 3]:295-297 DOI 10.1007/s10039-009-1580-4

Online publiziert: 10. Januar 2010

(c) Springer-Verlag 2010

\author{
R. Simon \\ BG-Unfallklinik Ludwigshafen
}

\title{
Bildgebende Diagnostik im Bereich der Hand
}

\section{Was leistet was?}

\section{Indikationen zur bildgebende Diagnostik}

Eine radiologische Diagnostik ist indiziert bei:

- Trauma,

- posttraumatischen Veränderungen (Pseudarthrose, Nekrose usw.), angefertigt - eine Aufnahme der rechten Hand von Frau Röntgen. Aus Anlass des 100-jährigen Jubiläums nach Entdeckung der Röntgenstrahlen wurde 1995 eine Sonderbriefmarke der Deutschen Bundespost herausgegeben, auf der ebenfalls eine Röntgenaufnahme der Hand abgebildet ist.

Die bildgebenden Verfahren im Bereich des Handgelenkes bzw. der Hand beinhalten schwerpunktmäßig konventionelle Röntgenaufnahmen (einschließlich Spezialaufnahmen), die Computertomographie (CT) und die Magnetresonanztomographie (MRT). Weitere bildgebende diagnostische Verfahren sind die Sonographie, Szintigraphie (Nuklearmedizin) und invasive Techniken (Arthrographie, Angiographie). Im folgenden Beitrag soll auf die „klassischen 3 Säulen“, Röntgendiagnostik - CT - MRT, näher eingegangen werden.

In einer unfallchirurgisch-orthopädischen Klinik der Maximalversorgung mit großer handchirurgischer Ambulanz und entsprechendem handchirurgischem Spektrum werden praktisch rund um die Uhr bildgebende Verfahren für die Diagnostik eingesetzt.
- Raumforderung,

- entzündlichen Prozessen,

- degenerativen Veränderungen und

- Missbildungen/Anomalien (Normvarianten).

Im Folgenden wird anhand dieses Indikationsspektrums beispielhaft der Stellenwert der bildgebenden Verfahren dargestellt.

\section{Trauma}

Beim frischen Trauma besitzt nach wie vor die konventionelle Röntgendiagnostik den höchsten Stellenwert. Frakturen können zuverlässig diagnostiziert und klassifiziert werden.

Bei speziellen Fragestellungen können mit Hilfe der Computertomographie (CT) wichtige Informationen zum Ausmaß der Fraktur und zur Gelenkbeteiligung und stellung erhalten werden.

Vorteile der Magnetresonanztomographie (MRT) beim frischen Trauma sind die Detektion von okkulten Frakturen und die Darstellung möglicher paraossärer und periartikulärer Weichteilverletzungen. Ein Beispiel hierfür ist die Diagnostik einer röntgenologisch okkulten Skaphoidfraktur bei entsprechender kli- nischer Symptomatik und negativem Röntgenbefund.

\section{Posttraumatische Veränderungen}

Eines der häufigsten Beispiele hierfür in unserer Klinik ist die Skaphoidpseudarthrose, die primär ebenfalls mit konventionellen Röntgenaufnahmen diagnostiziert wird.

Eine exakte Stadieneinteilung der Pseudarthrose ist mittels CT möglich. So können wesentlich genauer als mit den konventionellen Röntgenaufnahmen Resorptionszonen im Bereich des Pseudarthrosenspalts, zystische Veränderungen, Sekundärreaktionen mit Sklerosierung und Fragmentierung sowie die periskaphoidale Arthrose dargestellt werden.

Der diagnostische Gewinn der MRT besteht v. a. darin, eine Aussage über die Durchblutung bzw. Vitalität treffen zu können. So können mit der kontrastmittelgestützten MRT zuverlässig Perfusionsstörungen bis hin zur avitalen Knochennekrose diagnostiziert werden.

\section{Raumforderung}

Knochentumoren. Ihre Diagnose wird primär mittels konventionellen Röntgenuntersuchungen gestellt. Mit sorgfältiger Analyse des Röntgenbildes ist es in der Regel gut möglich, die Differenzialdiagnose einzugrenzen.

Im Einzelfall kann die CT bei der genaueren Beurteilung der Tumormorphologie hilfreich sein.

Bei Verdacht auf Malignität ist eine MRT-Untersuchung grundsätzlich sinn- 
Trauma Berufskrankh 2010 - 12[Suppl 3]:295-297 DOI 10.1007/s10039-009-1580-4

(c) Springer-Verlag 2010

\section{R. Simon \\ Bildgebende Diagnostik im Bereich der Hand. Was leistet was?}

\section{Zusammenfassung}

Röntgenaufnahmen, Computertomographie (CT) und Magnetresonanztomographie (MRT) stellen die „klassischen 3 Säulen" der bildgebenden Diagnostik des Handgelenks und der Hand dar. Eine radiologische Diagnostik ist indiziert bei Trauma, posttraumatischen Veränderungen, Raumforderungen, entzündlichen Prozessen, degenerativen Veränderungen und Missbildungen/Anomalien (Normvarianten). Bei sorgfältiger Indikationsstellung, exakter Lagerung des Patienten und optimaler technischer Einstellung können anhand von Röntgenübersichts- und -spezialaufnahmen bereits eine hohe Anzahl korrekter Diagnosen gestellt werden. Mit moder- nen CT-Techniken können hochauflösende dünne Schichtaufnahmen in allen Körperebenen multiplanar angefertigt bzw. rekonstruiert werden. Mit der MRT können zusätzlich auch Weichteilstrukturen dargestellt werden. Für den optimalen Einsatz der diagnostischen Verfahren ist der ständige Dialog zwischen den behandelnden Ärzten und den Radiologen von großer Bedeutung.

\section{Schlüsselwörter}

Hand · Handgelenk · Röntgenaufnahmen . Computertomographie $\cdot$ Magnetresonanztomographie

\section{Diagnostic imaging of the hand. What does what?}

\section{Abstract}

X-ray, computed tomography (CT) and magnetic resonance imaging (MRI) form the three classic pillars of diagnostic imaging of the wrist and hand. Diagnostic imaging is indicated in the case of post-traumatic changes, invasive and inflammatory processes, degenerative changes and deformities/anomalies (normal variants). In the case of correct indication, accurate patient positioning and optimal technical settings, a high rate of correct diagnoses can already be obtained from $\mathrm{X}$-rays and special views. Using modern CT techniques, high-resolution thin-slice images of all body areas can be used for multiplanar reformatting or reconstruction. MRI is additionally able to visualize soft-tissue structures. To optimize the use of diagnostic procedures, constant dialogue between clinics and radiologists is of great importance.

\section{Keywords}

Hand - Wrist · X-ray - Computed tomography . Magnetic resonance imaging voll, da zusätzlich zur Röntgen- und CTDiagnostik die Gewebecharakteristik des Tumors und die Vaskularisation beurteilt werden können. Zusätzlich kann mittels MRT die intraossäre Tumorausdehnung exakt dargestellt werden. Auch die Frage einer Tumorausdehnung in die Weichteilstrukturen kann mittels MRT zuverlässig beantwortet werden.

Weichteiltumoren. Neben der Sonographie besitzt hier die MRT herausragende Bedeutung. Wie auch bei Knochentumoren sind mit ihr Aussage zur genauen Tumorgröße und Lokalisation, Gewebebeschaffenheit und Vaskularisation möglich.

\section{Entzündliche Prozesse}

Osteitis/Osteomyelitis. Je nach Lokalisation und Ausdehnung eines entzündlichen Prozesses werden in der Regel sowohl konventionelle Röntgenaufnahmen als auch MRT-Aufnahmen (mit Kontrastmittel) benötigt. Bei osteodestruktiven Prozessen ist $u$. U. ergänzend auch eine CT-Diagnostik erforderlich, um das Ausmaß der knöchernen Destruktionen besser beurteilen und operative Maßnahmen exakt planen zu können.

Weichteilinfekt. Neben der klinischen und sonographischen Diagnose hat die MRT-Diagnostik den größten Stellenwert. Außer der genauen Lokalisation und Ausdehnung entzündlicher Veränderungen können mittels Kontrastmittelgabe zuverlässig einschmelzenden Prozesse diagnostiziert werden (Abszess, Phlegmone).

\section{Entzündlich-degenerative Erkrankungen}

Hierzu gehören v. a.:

- Gelenkveränderungen (Osteoarthropathien, Arthrose/Arthritis),

- Erkrankungen aus dem rheumatischen Formenkreis,

- Enthesiopathien und

- Systemerkrankungen.

Bei vielen dieser Fragestellungen sind in der Regel konventionelle Röntgenaufnahmen ausreichend. Bei Osteoarthropathien können mit Hilfe der CT die Gelenkver- 
hältnisse exakt und überlagerungsfrei dargestellt werden. Bei Erkrankungen aus dem rheumatischen Formenkreis im Frühstadium können beginnende Veränderungen mit der kontrastmittelgestützten MRT erkannt werden. Zu diesem Zeitpunkt sind röntgenologisch oft noch keine pathologischen Befunde sichtbar.

\section{Fehlbildungen und Deformitäten - Normvarianten}

Je nach klinischem Befund und Verdachtsdiagnose werden primär konventionelle Röntgenaufnahmen angefertigt. Bei unklarem Befund, insbesondere im Bereich der knöchernen Verhältnisse, kann ergänzend eine CT-Diagnostik sinnvoll sein.

\section{Stellenwert der einzelnen Verfahren}

Abschließend soll der Stellenwert der konventionellen Röntgendiagnostik, der Computertomographie und der Magnetresonanztomographie hervorgehoben werden.

\section{Konventionelle Röntgendiagnostik}

Mit exakter Lagerung des Patienten und optimaler technischer Einstellung können anhand der zur Verfügung stehenden Röntgenübersichts- und -spezialaufnahmen bereits eine hohe Anzahl korrekter Diagnosen gestellt werden. Entscheidend hierfür ist allerdings auch die korrekte Indikationsstellung durch den behandelnden Arzt, sodass je nach Fragestellung die „richtigen Röntgenaufnahmen“ angefertigt werden.

\section{Computertomographie}

Mittels der modernen Mehrschicht-Spiral-CT können hochauflösende dünne Schichtaufnahmen in allen Körperebenen multiplanar angefertigt bzw. rekonstruiert werden. Beispiel hierfür sind schrägsagittale Rekonstruktionen entlang der Skaphoidlängsachse zur exakten Beurteilung einer Skaphoidfraktur oder Pseudarthrose. Beispiele für axiale Rekonstruktionen sind die skapholunäre Dissoziation oder die Beurteilung des distalen Radioulnargelenks (DRUG).

\section{Magnetresonanztomographie}

Der große Vorteil der MRT gegenüber der konventionellen Röntgendiagnostik und der CT ist die Darstellung der knöchernen und Weichteilstrukturen. So können beispielsweise Weichteilverletzungen, z. B. im Bereich der Sehnen- und Bandstrukturen, zuverlässig diagnostiziert werden. Auch andere Weichteilprozesse (z. B. Ganglion, Tumor) sind eine Domäne der MRT, die eine exakte Therapie- bzw. Operationsplanung ermöglicht.

Der Stellenwert der kontrastmittelgestützten MRT zur Beurteilung der Vaskularisation bei entzündlichen Prozessen, Tumoren und Osteonekrosen wurde bereits hervorgehoben.

\section{Fazit}

Die bildgebende Diagnostik wurde seit der weltweit ersten Röntgenaufnahme der Hand kontinuierlich weiterentwickelt. Neben der konventionellen Röntgendiagnostik spielen die modernen Schnittbildverfahren (CT, MRT) eine herausragende Rolle. Grundlage für die Wahl der „richtigen“ bildgebenden Modalität sind eine exakte Anamnese und klinische Untersuchung des Patienten. Für den optimalen Einsatz der diagnostischen Verfahren ist der ständige Dialog zwischen behandelnden Ärzten und Radiologen von großer Bedeutung.

\section{Korrespondenzadresse}

\section{Dr. R. Simon}

BG-Unfallklinik Ludwigshafen,

Ludwig-Guttmann-Straße 13,

67071 Ludwigshafen

simon@bgu-ludwigshafen.de

Interessenkonflikt. Der korrespondierende Autor gibt an, dass kein Interessenkonflikt besteht. 